\title{
Structure and spectrum of two-dimensional clusters confined in a hard wall potential
}

\author{
Minghui Kong, B. Partoens, A. Matulis, ${ }^{*}$ and F. M. Peeters ${ }^{\dagger}$ \\ Departement Natuurkunde, Universiteit Antwerpen (Campus Drie Eiken), Universiteitsplein 1, B-2610 Antwerpen, Belgium
}

(Received 17 September 2003; revised manuscript received 26 November 2003; published 31 March 2004)

\begin{abstract}
The structural and dynamical properties of two-dimensional (2D) clusters of equally charged classical particles, which are confined in an external hard wall potential, are investigated through the Monte Carlo simulation technique. The ground-state configuration is investigated as a function of the interparticle interaction (Coulomb, dipole, logarithmic, and screened Coulomb). The excitation spectrum corresponding to the ground-state configuration of the system is discussed. The eigenmodes are investigated and the corresponding divergence and rotor are calculated, which indicates the "shearlike" and "compressionlike" aspects of the different modes. Both small and large clusters are considered.
\end{abstract}

DOI: 10.1103/PhysRevE.69.036412

PACS number(s): 52.27.Lw, 36.40.Sx, 82.70.Dd

\section{INTRODUCTION}

In 1934, Wigner predicted [1] that electrons crystallize and form a lattice if the density of the three-dimensional (3D) electron gas is lowered beyond a certain critical value. Such Wigner crystals have been observed in 2D systems, such as electrons on the surface of liquid helium [2], electrons in quantum dots [3], colloidal suspensions [4], and in confined plasma crystals [5]. When the classical Wigner crystal forms, the Coulomb interaction potential is much larger than the thermal energy, which is a necessary requirement for the system to self-organize into an ordered condensed phase.

Recently, such Wigner-like ordering has been investigated in systems consisting of a finite number of particles. Most of such studies focus on systems with an external parabolic confinement, which is similar to the action of a uniform neutralizing background of charges. Other type of confinements which have also been considered are those residing from a fixed positive charge. Such a positive charge keeps the repelling charged particles together, and is in fact the classical analog for atoms [6,7]. An extreme case of confinement is the one consisting of hard walls. Interesting reentrant melting behavior was observed for colloidal particles dissolved in water $[8,9]$ in such a hard wall potential, which is absent in a parabolic confined system. The radial particle fluctuations are responsible for an enhanced locking of adjacent shells [10]. In fact the short-range interparticle interaction in combination with the hard wall confinement leads to the unusual reentrant behavior in its orientational order. In Refs. [10,11] the melting of some specific clusters was investigated theoretically, but no systematic study of the ground state and normal modes was made. Such a study will be presented here, which is further motivated by the fact that in a hard wall confined system the physics is very different, e.g., (1) there is a very inhomogeneous distribution of particles because many of them are pushed to the edge of the system where they create a nonparabolic confinement potential for

\footnotetext{
*Permanent address: Semiconductor Physics Institute, Goštauto 11, 2600 Vilnius, Lithuania.

†Electronic address: francois.peeters@ua.ac.be
}

the other particles; and (2) the average particle density is unaltered with varying interparticle interaction which is different for parabolic confinement where the density increases with decreasing interparticle interaction strength.

The purpose of this work is to present a detailed study of the ground-state properties of $2 \mathrm{D}$ clusters with different interparticle interactions (Coulomb, dipole, logarithmic, and screened Coulomb, i.e., the Yukawa potential), which are laterally confined in a hard wall potential. Both small and large systems will be discussed. The excitation spectrum corresponding to the ground-state configuration and the corresponding divergence and rotor of it, which describe the amount of "shearlike" and "compressionlike" nature of the modes, are investigated.

The present paper is organized as follows. In Sec. II, the model system and the numerical approach are described. Sec. III is devoted to the ground-state configuration with different interparticle interactions. In Sec. IV, the eigenmode spectrum for these clusters is discussed, and the shearlike and compressionlike character of the different modes are shown in Sec. V. Our conclusions are given in Sec. VI. The dependence of the normal mode frequencies on the steepness of the confinement potential is discussed in Appendix A for the two-particle system. Appendix B gives an analysis of the radial motion of particles situated at the border of the hard wall confinement potential.

\section{NUMERICAL APPROACH}

The potential energy of a 2D system of $N$ charged particles in a hard wall confinement potential and interacting through a repulsive $1 / r^{n^{\prime}}$ potential is given by

$$
E=\sum_{i=1}^{N} V\left(r_{i}\right)+\frac{e^{2}}{\varepsilon R} \sum_{i>j}^{N} \frac{R^{n^{\prime}}}{\left|\vec{r}_{i}-\vec{r}_{j}\right|^{n^{\prime}}},
$$

with the hard wall potential

$$
V(r)= \begin{cases}0 & \text { for } r<R \\ \infty & \text { for } r \geqslant R\end{cases}
$$


and $R$ the radius of the hard wall, $e$ the particle charge, $\varepsilon$ the dielectric constant of the medium the particles are moving in, and $\vec{r}_{i}=\left(x_{i}, y_{i}\right)$ the position of the $i$ th particle with $r_{i}$ $\equiv\left|\vec{r}_{i}\right|$.

As the above potential energy depends only on two parameters which depend on the experimental setup (namely, $R$ and $e^{2} / \varepsilon$ ) and we have to choose also two units (namely, for the length and the energy) it is possible to scale out all experimental dependent parameters. For the natural choice of $r_{0}=R$ as unit of length and $E_{0}=\left(e^{2} / \varepsilon\right) / R$ as unit of energy, the potential energy in dimensionless form becomes

$$
E=\sum_{i=1}^{N} V\left(r_{i}\right)+\sum_{i>j}^{N} \frac{1}{\left|\vec{r}_{i}-\vec{r}_{j}\right|^{n^{\prime}}},
$$

with the hard wall potential

$$
V(r)= \begin{cases}0 & \text { for } r<1 \\ \infty & \text { for } r \geqslant 1 .\end{cases}
$$

Note that this Hamiltonian only depends on the number of particles $N$ and not on the radius of the hard wall.

In the present paper, we mainly present results for particles interacting through the Coulomb potential (i.e., $n^{\prime}$ $=1)$. We also consider other interactions such as $1 / r_{i j}^{3}$, $\exp \left(-r_{i j} / \lambda_{D}\right) / r_{i j}=\exp \left[-\left(r_{i j} / R\right)\left(R / \lambda_{D}\right)\right] / r_{i j}=\exp \left(-\kappa r_{i j} / R\right) / r_{i j}$ $\left(\lambda_{D}\right.$ is the screening length and $\kappa=R / \lambda_{D}$ ) and $-\ln r_{i j}$ for the cases of dipole, screened Coulomb, and logarithmic interparticle interaction, respectively $\left(r_{i j} \equiv\left|\vec{r}_{i}-\vec{r}_{j}\right|\right)$.

The Monte Carlo simulation technique [12] extended with a gradient method is used to obtain the ground state configuration. The eigenmode frequencies are obtained from the eigenvalues of the dynamical matrix [13]

$$
E_{\alpha \beta, i j}=\left.\frac{\partial^{2} E}{\partial r_{\alpha, i} \partial r_{\beta, j}}\right|_{r_{\alpha, i}=r_{\alpha, i}^{n}},
$$

where $\left\{r_{\alpha, i}^{n}\right\}$ is the ground-state configuration. The eigenfrequencies in this paper are expressed in the unit $\omega^{\prime}$ $=\sqrt{E_{0} /\left(m r_{0}^{2}\right)}$.

A special situation arises for the outer-shell particles as they can only move along the perimeter, and radial outward movement of the outer-shell particles is forbidden. Therefore it is convenient to diagonalize the dynamical matrix expressed in polar coordinates. The oscillations of the particles around their equilibrium positions have a component along the $\rho$ direction and one along the $\theta$ direction. In the hard wall limit, the particles in the outer-shell can have only one component, namely, along the $\theta$ direction, and we neglect the eigenmodes along the $\rho$ direction, since the corresponding eigenfrequencies are infinite.

As an illustration we present in Appendix A the analytical results of the two-particle problem for a general $r^{n}$ confinement potential.

\section{GROUND-STATE CONFIGURATIONS}

Unlike for the parabolic confinement case with Coulomb interparticle interactions, the particles are not attracted to the center of the confinement potential but they repel each other such that they move to the edge of the system. In this case, the previously [11] found simple polygons are formed at the edge. For a cluster with a small number of particles, one concentric shell at the edge will form and the center region will be empty, while for a large number of particles the center region will resemble a triangular lattice, however the shell structure stays more pronounced than for the parabolic case.

Table I shows the packing sequences of the ground state for $N=2,3, \ldots, 50$ with different interparticle interactions, including the Coulomb, dipole, and screened Coulomb (for $\kappa=0.5,2.0,4.0)$ cases. The configuration is indicated by the number of particles in the different rings. The energy per particle $E / N$ is also given. This table is rather exhaustive and should be compared with a similar one published in Refs. $[11,14]$ for the parabolic confinement potential.

First, we discuss the case for Coulombic interparticle interaction. From Table I, we can see how shells are filled by the particles and new shells appear. For small $N \in\{2-11\}$, the particles are situated at the edge of the wall. When a critical number of particles at the edge is achieved, a new shell appears. This occurs for $N=12$ and is, as said above, independent of the radius of the confinement potential. From $N \in\{12-29\}$, the system has a two-shell structure. One interesting phenomenon can be observed in such a hard wall case. When there are two shells, the edge is always the preferable position for the new particles. New particles will begin to join into the center shell only if the edge reaches a critical number of particles. When the inner ring has six particles a third ring is created. This occurs for $N \in\{30-50\}$. Note that this is different from the parabolic confinement case where a new shell forms when the inner ring is filled with five particles.

It was shown in Ref. [14] that for a system with parabolic confinement the configurations do not differ very strongly for short- or long-range interactions, contrary to what we find for the hard wall case. This is nicely illustrated in Table I if we compare the dipole and Yukawa (with different screening lengths) with the Coulomb case. Note also that the configuration for $N=29$ in the case of dipole interaction, namely, $(3,9,17)$, corresponds with the experimentally observed configuration in Refs. [8,9]. For the Yukawa system, the structure is strongly dependent on the screening strength $\kappa$. For a small value of $\kappa=0.5$ the structure of the system is quite similar to the pure Coulomb case (see the third and sixth columns in Table I). When increasing $\kappa$, one can see that more and more particles can appear in the center region. The table also shows that for more short-range interparticle interactions new shells are formed for lower $N$ values. This is not surprising because the interparticle interaction is diminished while the size of the system is not (in contrast to the parabolic case where the size of the system becomes smaller with increasing $\kappa[15,16])$.

We investigated in more detail how many particles can be placed at the edge before one of the particles is placed in the 
TABLE I. The ground state for $N=2,3 \ldots, 50$ particles confined in a 2D hard wall with Coulomb, dipole, and screened Coulomb interparticle interactions, respectively. The energies $(E / N)$ and the shell structures $\left(N_{1}, N_{2}, \ldots\right)$ of the ground-state configuration are shown.

\begin{tabular}{|c|c|c|c|c|c|c|c|}
\hline \multirow[b]{2}{*}{$\mathrm{N}$} & \multirow{2}{*}{$\begin{array}{c}\mathrm{E} / \mathrm{N} \\
(\text { Coulomb) }\end{array}$} & \multirow{2}{*}{$\begin{array}{l}\text { Configuration } \\
\text { (Coulomb) }\end{array}$} & \multirow{2}{*}{$\begin{array}{c}\mathrm{E} / \mathrm{N} \\
(\text { dipole })\end{array}$} & \multirow{2}{*}{$\begin{array}{l}\text { Configuration } \\
\text { (dipole) }\end{array}$} & \multicolumn{3}{|c|}{ Configuration (Yukawa) } \\
\hline & & & & & $\kappa=0.5$ & $\kappa=2.0$ & $\kappa=4.0$ \\
\hline 2 & 0.25000 & 2 & 0.06250 & 2 & 2 & 2 & 2 \\
\hline 3 & 0.57735 & 3 & 0.19245 & 3 & 3 & 3 & 3 \\
\hline 4 & 0.95711 & 4 & 0.41605 & 4 & 4 & 4 & 4 \\
\hline 5 & 1.37639 & 5 & 0.76085 & 5 & 5 & 5 & 5 \\
\hline 6 & 1.82735 & 6 & 1.25495 & 6 & 6 & 6 & 6 \\
\hline 7 & 2.30477 & 7 & 1.92680 & 7 & 7 & 7 & 1,6 \\
\hline 8 & 2.80487 & 8 & 2.56095 & 1,7 & 8 & 1,7 & 1,7 \\
\hline 9 & 3.32483 & 9 & 3.38223 & 1,8 & 9 & 1,8 & 1,8 \\
\hline 10 & 3.86245 & 10 & 4.42647 & 1,9 & 10 & 1,9 & 1,9 \\
\hline 11 & 4.41597 & 11 & 5.63037 & 2,9 & 1,10 & 1,10 & 2,9 \\
\hline 12 & 4.96464 & 1,11 & 6.84760 & 2,10 & 1,11 & 2,10 & 2,10 \\
\hline 13 & 5.52365 & 1,12 & 8.14532 & 3,10 & 1,12 & 2,11 & 3,10 \\
\hline 14 & 6.09624 & 1,13 & 9.55674 & 3,11 & 1,13 & 2,12 & 3,11 \\
\hline 15 & 6.68141 & 1,14 & 11.11835 & 4,11 & 1,14 & 3,12 & 4,11 \\
\hline 16 & 7.27826 & 1,15 & 12.73777 & 4,12 & 2,14 & 3,13 & 4,12 \\
\hline 17 & 7.87157 & 2,15 & 14.60145 & 5,12 & 2,15 & 4,13 & 5,12 \\
\hline 18 & 8.47100 & 2,16 & 16.44619 & 5,13 & 3,15 & 4,14 & 5,13 \\
\hline 19 & 9.07868 & 3,16 & 18.56347 & $1,5,13$ & 3,16 & 4,15 & 5,14 \\
\hline 20 & 9.68150 & 3,17 & 20.61187 & $1,6,13$ & 3,17 & 5,15 & $1,5,14$ \\
\hline 21 & 10.29425 & 3,18 & 22.62037 & $1,6,14$ & 4,17 & 5,16 & $1,6,14$ \\
\hline 22 & 10.91463 & 4,18 & 24.96092 & $1,6,15$ & 4,18 & $1,5,16$ & $1,6,15$ \\
\hline 23 & 11.53049 & 4,19 & 27.26438 & $1,7,15$ & 4,19 & $1,5,17$ & $1,7,15$ \\
\hline 24 & 12.15533 & 4,20 & 29.87944 & $1,7,16$ & 5,19 & $1,6,17$ & $1,7,16$ \\
\hline 25 & 12.78663 & 5,20 & 32.54436 & $1,8,16$ & 5,20 & $1,6,18$ & $1,7,17$ \\
\hline 26 & 13.41427 & 5,21 & 35.27964 & $2,8,16$ & 5,21 & $1,7,18$ & $1,8,17$ \\
\hline 27 & 14.05013 & 5,22 & 38.10941 & $2,8,17$ & 6,21 & $1,7,19$ & $2,8,17$ \\
\hline 28 & 14.69087 & 6,22 & 40.97470 & $3,8,17$ & $1,6,21$ & $1,7,20$ & $2,8,18$ \\
\hline 29 & 15.32924 & 6,23 & 43.81985 & $3,9,17$ & $1,6,22$ & $1,8,20$ & $3,8,18$ \\
\hline 30 & 15.96932 & $1,6,23$ & 46.82763 & $3,9,18$ & $1,6,23$ & $1,8,21$ & $3,9,18$ \\
\hline 31 & 16.61023 & $1,6,24$ & 50.03572 & $4,9,18$ & $1,7,23$ & $2,8,21$ & $3,9,19$ \\
\hline 32 & 17.25843 & $1,6,25$ & 53.20328 & $4,10,18$ & $1,7,24$ & $2,8,22$ & $3,9,20$ \\
\hline 33 & 17.90323 & $1,7,25$ & 56.45537 & $4,10,19$ & $1,7,25$ & $3,8,22$ & $4,9,20$ \\
\hline 34 & 18.55396 & $1,7,26$ & 59.96733 & $4,11,19$ & $1,8,25$ & $3,9,22$ & $4,10,20$ \\
\hline 35 & 19.20978 & $1,8,26$ & 63.51491 & $5,11,19$ & $1,8,26$ & $3,9,23$ & $4,10,21$ \\
\hline 36 & 19.86301 & $1,8,27$ & 67.00782 & $5,11,20$ & $2,8,26$ & $3,10,23$ & $4,11,21$ \\
\hline 37 & 20.52278 & $1,8,28$ & 70.81054 & $1,5,11,20$ & $2,8,27$ & $3,10,24$ & $5,11,21$ \\
\hline 38 & 21.18212 & $2,8,28$ & 74.53620 & $1,6,11,20$ & $2,9,27$ & $4,10,24$ & $5,11,22$ \\
\hline 39 & 21.84389 & $2,8,29$ & 78.22570 & $1,6,12,20$ & $2,9,28$ & $4,10,25$ & $1,5,11,22$ \\
\hline 40 & 22.50297 & $2,9,29$ & 81.89272 & $1,6,12,21$ & $3,9,28$ & $4,11,25$ & $1,5,11,23$ \\
\hline 41 & 23.16711 & $2,9,30$ & 85.98871 & $1,6,12,22$ & $3,9,29$ & $4,11,26$ & $1,6,11,23$ \\
\hline 42 & 23.82918 & $3,9,30$ & 90.01315 & $1,6,13,22$ & $3,10,29$ & $5,11,26$ & $1,6,12,23$ \\
\hline 43 & 24.49545 & $3,9,31$ & 94.05687 & $1,7,13,22$ & $3,10,30$ & $5,11,27$ & $1,6,12,24$ \\
\hline 44 & 25.16090 & $3,10,31$ & 98.43269 & $1,7,13,23$ & $4,10,30$ & $5,12,27$ & $1,6,13,24$ \\
\hline 45 & 25.82957 & $3,10,32$ & 102.7506 & $1,7,14,23$ & $4,10,31$ & $1,5,12,27$ & $1,6,13,25$ \\
\hline 46 & 26.49958 & $4,10,32$ & 107.3208 & $1,8,14,23$ & $4,11,31$ & $1,5,12,28$ & $1,7,13,25$ \\
\hline 47 & 27.17037 & $4,10,33$ & 111.7210 & $2,8,14,23$ & $4,11,32$ & $1,6,12,28$ & $1,7,13,26$ \\
\hline 48 & 27.84116 & $4,11,33$ & 116.2977 & $2,8,14,24$ & $5,11,32$ & $1,6,12,29$ & $1,7,14,26$ \\
\hline 49 & 28.51431 & $4,11,34$ & 120.9331 & $3,8,14,24$ & $5,11,33$ & $1,6,13,29$ & $1,8,14,26$ \\
\hline 50 & 29.19165 & $5,11,34$ & 125.5184 & $3,8,15,24$ & $5,12,33$ & $1,6,13,30$ & $2,8,14,26$ \\
\hline
\end{tabular}




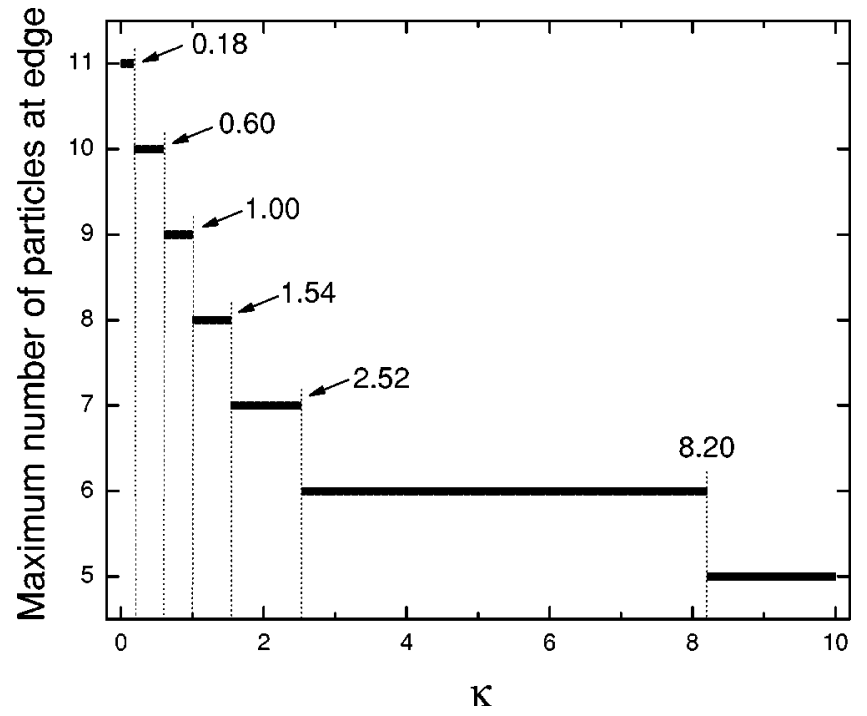

FIG. 1. The maximum number of particles at the edge of the hard wall confinement before one particle is situated in the center as a function of the interaction strength $\kappa$.

center, in case of a screened Coulomb interparticle interaction. The energy of the configuration with $N$ particles equidistantly placed on a single ring at the edge is given by

$$
E_{\bigcirc}=\sum_{i=1}^{N-1} \sum_{j=i+1}^{N} \frac{e^{-2 \kappa|\sin [\pi(i-j) / N]|}}{2\left|\sin \frac{\pi(i-j)}{N}\right|},
$$

and the energy of $N-1$ particles at the edge with 1 in the center is given by

$$
E_{\odot}=\sum_{i=1}^{N-2} \sum_{j=i+1}^{N-1} \frac{e^{-2 \kappa|\sin [\pi(i-j) /(N-1)]|}}{2\left|\sin \frac{\pi(i-j)}{N-1}\right|}+(N-1) e^{-\kappa}
$$

The maximum value of $N$ for which $E_{\odot}<E_{\odot}$ is the maximum number of particles at the edge. This value is shown in Fig. 1 as a function of $\kappa$. For $\kappa>8.2$ the sixth particle always goes to the center.

Another pronounced difference in the configuration for a Yukawa system can be observed. In the parabolic case, the ground-state configuration changes from a shell-like structure into a hexagonal lattice with increasing $\kappa[15,16]$. For hard wall confinement the radius of the system does not change and the configuration stays shell-like, even up to large $\kappa$ values.

The case with logarithmic interparticle interaction was considered as well. We did not add the result to Table I, since the configuration is always a single shell, which is a consequence of Earnshaw's theorem $[17,18]$ in two dimensions. The interaction potential is long range, and all particles are situated at the edge of the hard wall.

For large systems the configuration is determined by two competing effects, namely, circular symmetry and triangular structure (Wigner lattice). The radial distributions together

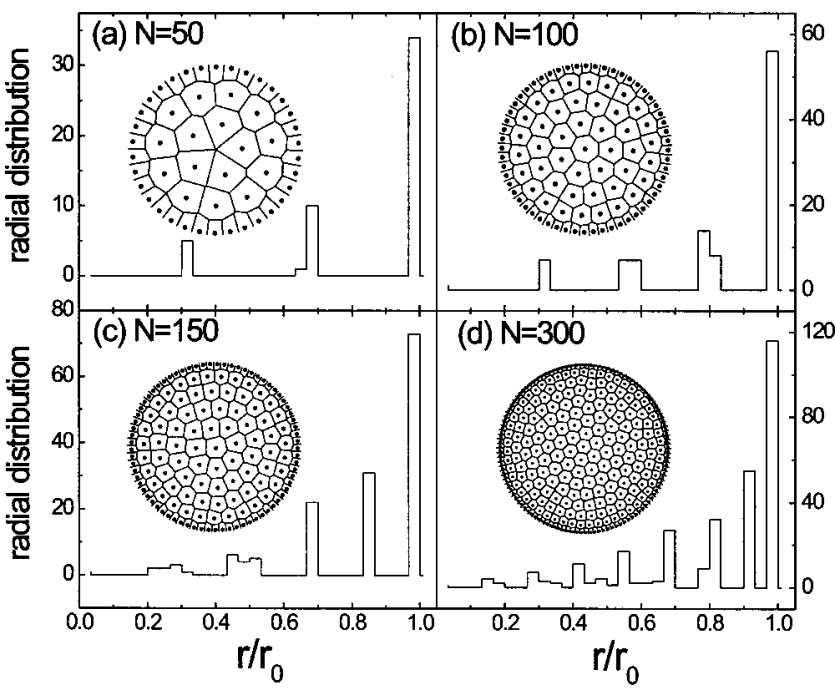

FIG. 2. The radial distribution for systems with $N=50,100,150$, and 300 particles. The insets show the configurations and their Voronoi structures.

with the configurations for $N=50,100,150$, and 300 are shown in Fig. 2 and this shows that for 50, 100 and 150 particles a clear shell structure is present. For $N=50$ particles [see Fig. 2(a)], there are three shells and the configuration is $(5,11,34)$. For $N=100$ particles [see Fig. 2(b)], one particle is situated in the center with four shells around it with the configuration $(1,7,14,22,56)$, and for $N=150$ [see Fig. 2(c) with configuration $(1,8,15,22,31,73)]$ one more ring is present. Figure 2(d) shows the configuration for $N$ $=300$. Notice that only the outer shells have a well-defined radius while the inner shells have no clear radius, reflecting that the inner region has a more triangular-lattice-like structure which can also be seen from the Voronoi construction in the inset of Fig. 2(d). In the parabolic case (and for Coulombic interaction), a triangular structure starts to appear in the center already for about 100 particles $[11,19]$, while for the hard-wall system a triangular lattice appears in the central region for $N \gtrsim 300$. Also the outer-shell structure is more pronounced than for parabolic confinement. Note however that in our hard wall system many particles are situated at the edge, for example, for the $N=300$ system the outer ring consists of $N_{E}=116$ particles. By excluding these edge particles, the triangular lattice in the center starts to appear from $300-N_{E}=184$, which is already closer to but still larger than for the parabolic confinement case.

It was shown before that the topological charge in case of a parabolically confined system is always -6 and that the position of the defects for large systems is in the transition region between the outer rings and the central triangular lattice [19]. The number of defects for the hard wall system is much larger, and are now also situated near the edge, but still the total topological charge always equals -6 .

On each shell, the particles are equally distributed. Unlike the parabolic confinement case with Coulomb interaction, for hard wall confinement the interparticle spacing in a shell decreases with increasing shell number. Those results are shown in Fig. 3(a). The inset in Fig. 3(a) shows the distance between shells for $N=50,100,150$, and 300 particles. One 

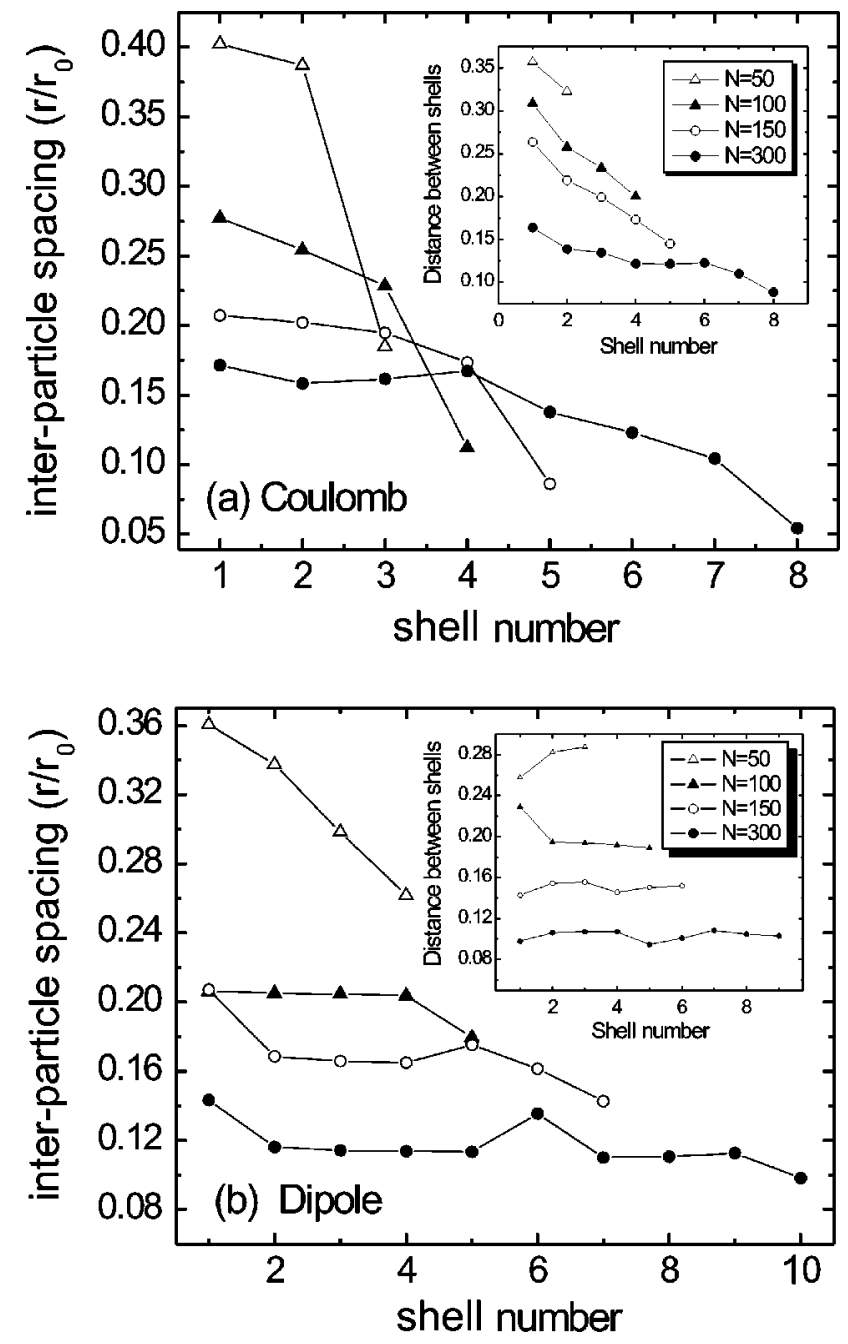

FIG. 3. The interparticle spacing within the shell as a function of the shell number for $N=50,100,150$, and 300 particles interacting through the (a) Coulomb and (b) dipole interaction potential for hard wall confinement. The insets show the distance between shells as a function of the shell number.

can see that the distance between shells always decreases from the inner shell to the outer shell. Both effects together make the density increase from the center to the edge, in contrast to the parabolic confinement case [11].

It is interesting to compare the results for our hard wall system with Coulomb interacting particles with a more shortrange interaction such as the dipole interparticle potential. Figure 3(b) shows the interparticle spacing in the different shells for the dipole system. One can see that it does not depend strongly on the shell number. The same is true for the distance between the shells, as shown in the inset of Fig. 3(b). As a result, the particles are more uniformly distributed in the case of the short-range dipole interaction than for the long-range Coulomb interaction. This is again a consequence of the fact that for more short-range interparticle interactions, particles appear in the center at lower $N$ values.

\section{THE EIGENMODE SPECTRUM}

In this section, we discuss the excitation spectrum of the ground-state configuration of the Coulomb system with a

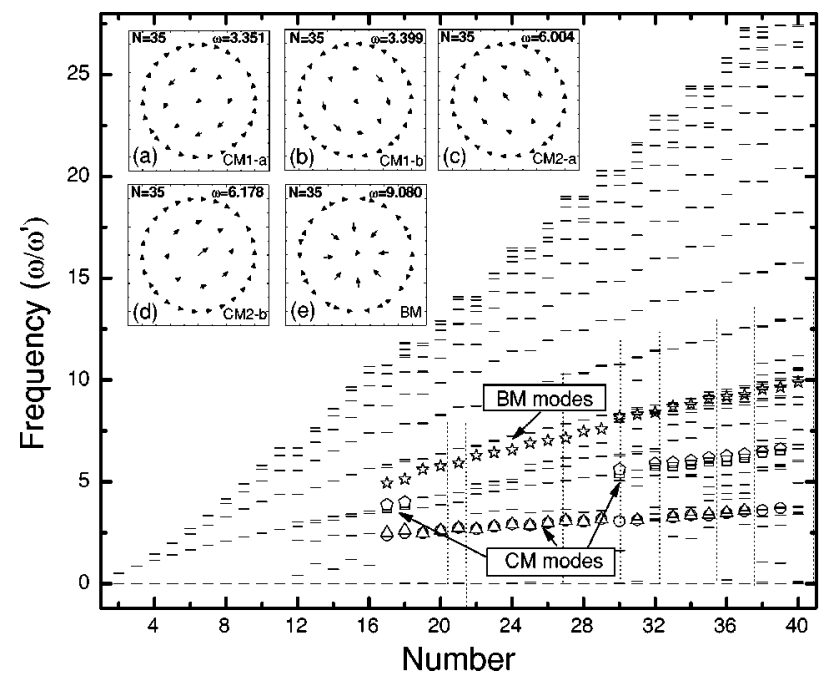

FIG. 4. Excitation spectrum of normal modes as a function of the number of particles for $N=2-40$ for the hard wall confinement. The CM-like and breathing-like modes are marked by symbols. The insets show the four CM-like modes and the breathing-like mode for $N=35$.

hard wall confinement. The spectral properties of the groundstate configurations for parabolic confinement were presented in Ref. [13]. The excitation of the normal modes of a 2D Coulomb clusters in laboratory complex plasmas was recently observed $[20,21]$. All the previous theoretical calculations and experimental results are for systems with a parabolic confinement.

Since the particles at the edge are practically frozen at the wall, they can only move along the perimeter. A detailed analysis of the radial motion of the particles situated at the edge of the hard wall is given in Appendix B. Notice that the number of particles at the edge is a considerable part of the whole system. There are $2 N$ normal modes for an $N$-particle system. In the hard wall case, the radial motion of particles at the edge has a large energy and consequently the number of relevant modes of the hard wall system is $2 N-N_{E}$, with $N_{E}$ the total number of particles at the edge. We plot the excitation spectrum of the normal modes as a function of the number of particles for hard wall confinement and Coulomb interparticle interaction in Fig. 4. For the parabolic confined system, there are three eigenfrequencies which are independent of $N[13]: \omega=0, \sqrt{2}$, and $\sqrt{6}$, which correspond to the rotation of the system as a whole, the center-of-mass mode $(\mathrm{CM})$, and the breathing mode $(\mathrm{BM})$, respectively. In the hard wall case only the $\omega=0$ frequency is still present. For $N=2-11$, the particles are all situated at the edge, and there is no sense of discussing the CM or BM frequency for hard wall confinement. Starting from $N>16$, more than one particle is situated in the central region. It is then possible to assign approximately those two eigenmodes (i.e., the CM and BM mode) to some of the eigenmodes of the particles in the central region. Examples of the CM-like and breathinglike modes for $N=35$ are shown in the insets of Fig. 4, and Figs. 6(g) and 6(h) show the corresponding CM-like and breathinglike modes for the central particles for $N=100$, respectively. While for the parabolic case, the amplitudes for 


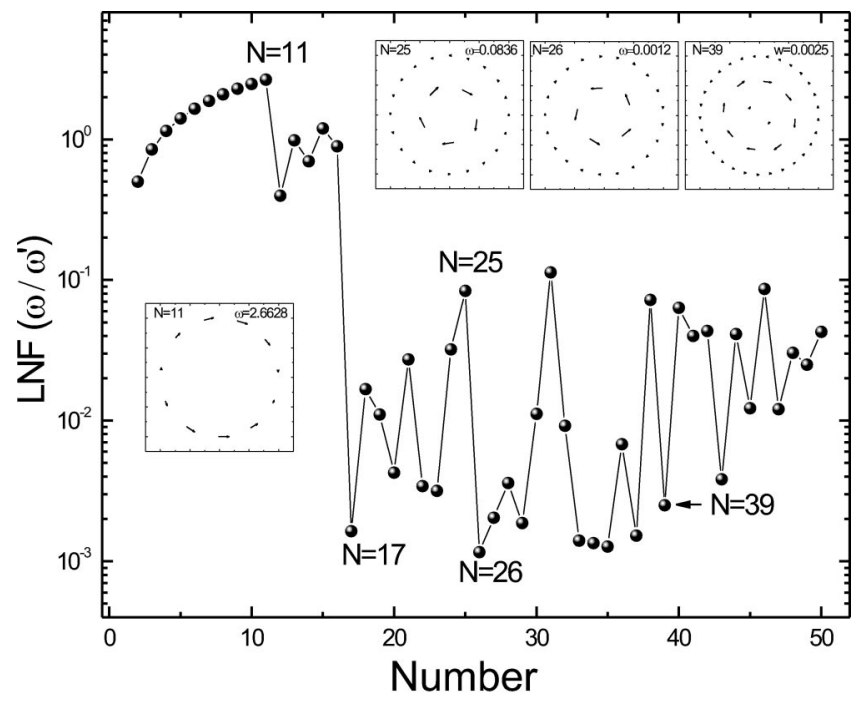

FIG. 5. The lowest nonzero eigenfrequency for $N=2, \ldots, 50$. The insets show the corresponding eigenmodes for $N=11,25,26$, and 39.

all particles are equal, now the particles which are closest to the center have the largest amplitudes. The frequencies corresponding with these $\mathrm{CM}$-like and breathinglike modes of the central particles are marked in Fig. 4 by symbols. Note that the degeneracy of the CM-like modes is slightly lifted and that for some clusters it is possible to find four CM-like modes. Comparing Fig. 4(b) with Fig. 4(c) one can see that in these cases the direction of the motion of the outer particles with respect to the direction of the motion of the central particles is different.

It was previously found that for small Coulomb systems, the lowest nonzero frequency mode (LNF) corresponds to intershell rotation, whereas for larger number of particles it corresponds to the excitation of a vortex-antivortex pair [13]. This is not the case anymore for the hard wall system. Figure 5 shows the LNF up to $N=50$ particles. When there is more than one particle at the center, the LNF mode corresponds always, also for large systems, with the rotation of all central particles as a whole in the direction opposite to the one for the particles at the edge [see insets of Fig. 5 for $N=25,26$, and 39 and Fig. 6(b) for $N=100$ ]. For $N<12$ all particles are at the edge and the LNF modes are similar to the one shown for $N=11$ in the inset of Fig. 5, namely, the two halves of the wide ring move in opposite direction. The reason why the LNF for $N=25$ [configuration $(5,20)]$ and $N=26$ [configuration $(5,21)]$ differ by two orders of magnitude is similar as for the parabolic confinement system [13]: for $N=25$ the number of particles in the outer shell is a multiple of the number of particles in the inner shell, resulting in a more stable configuration against intershell rotation (i.e., due to a commensurability effect), and $N=25$ corresponds to magic number clusters. Most of the other peaks in Fig. 5 can be explained in a similar way.

As in the system with $N=100$ particles in a hard wall confinement, most particles are situated at the edge, and therefore it may be wrong to consider it already as a large system. Therefore we also looked at the LNF of a larger

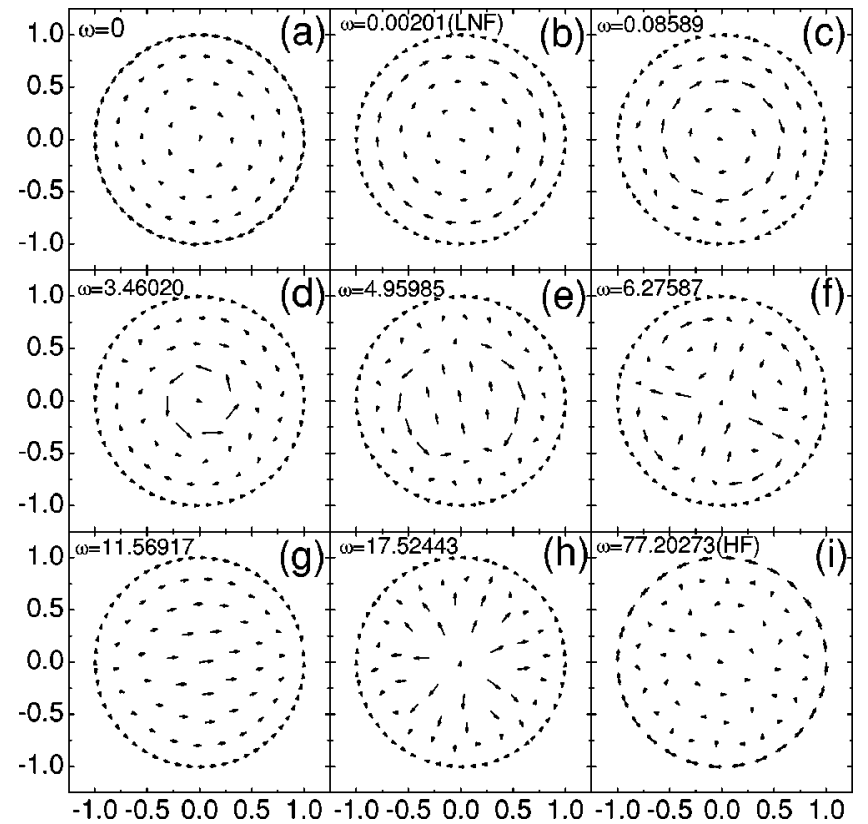

FIG. 6. Some eigenvectors for the cluster with $N=100$ particles.

system, namely, for $N=300$. Still the LNF corresponds with the rotation of the central particles as a whole and the vortexantivortex motion appears only for higher frequencies.

It is also interesting to have a look at the higher excited modes as they are much more easy to interpret than in the parabolic case. Figure 6 shows nine modes for $N=100$. With increasing frequency, after the rotation of the cluster as a whole [Fig. 6(a)] and the rotation of the central particles as a whole [Fig. 6(b)], particles with larger amplitudes appear close to the center of the system, resulting in many different types of intershell rotations [Figs. 6(c) and 6(d)]. Higher excitations may consist of a vortex-antivortex pair, or multiples of such pairs [Figs. 6(e) and 6(f)]. The highest frequency mode for parabolic confinement was discussed in Refs. $[19,21]$. In these modes the inner particles have larger amplitudes than the outer particles. We found the opposite behavior in the hard wall case. In Fig. 6(i), one can see the largest amplitude for the particles at the edge where the nearest particles at the edge move in opposite directions which is typical for the case of an optical mode.

\section{PROPERTIES OF THE NORMAL MODES}

In previous results for a 2D classical infinite system [22], two types of waves with dispersion relations $\omega \approx k$ (lateral sound waves) and $\omega \approx \sqrt{k}$ (longitudinal plasma waves), were observed. The latter one arises from the long-range nature of the Coulomb interaction [23]. These waves are related to the compressionlike and shearlike modes observed in finite confined cluster. The compressional and shearlike properties can be extracted from the divergence and rotor of the velocity field, respectively. In this paper, we will associate a single number to the shearlike and compressionlike character of the different modes by calculating the spatial average of the square of the divergence $\vec{\nabla} \cdot \vec{v}$ and the vorticity $(\vec{\nabla} \times \vec{v})_{z}$ of the velocity field, following the approach of Ref. [13]. 
The $z$ components of the rotor $\psi_{r}(k)=\vec{e}_{z} \cdot \operatorname{rot} \psi(k)$ and the divergence $\psi_{d}(k)=\operatorname{div} \psi(k)$ of the field of eigenvectors of mode $k$ are

$$
\begin{aligned}
& \psi_{d}(k)=\frac{1}{N} \sum_{i=1}^{N} \psi_{d, i}^{2}(k), \\
& \psi_{r}(k)=\frac{1}{N} \sum_{i=1}^{N} \psi_{r, i}^{2}(k),
\end{aligned}
$$

where the values of $\psi_{d, i}(k)$ and $\psi_{r, i}(k)$ for the $i$ th particle are given by

$$
\begin{gathered}
\psi_{d, i}(k)=\frac{1}{M} \sum_{m=1}^{M}\left(\vec{r}_{i}-\vec{r}_{m}\right) \cdot\left[\vec{A}_{i}(k)-\vec{A}_{m}(k)\right] /\left|\vec{r}_{i}-\vec{r}_{m}\right|^{2}, \\
\psi_{r, i}(k)=\frac{1}{M} \sum_{m=1}^{M}\left|\left(\vec{r}_{i}-\vec{r}_{m}\right) \times\left[\vec{A}_{i}(k)-\vec{A}_{m}(k)\right]\right| /\left|\vec{r}_{i}-\vec{r}_{m}\right|^{2},
\end{gathered}
$$

Here, $m$ and $M$ denote the index and number of neighboring particles of particle $i, \vec{r}_{m}$ is the positional coordinate of a neighboring particle, and $\vec{A}_{i}(k)$ is the eigenvector of particle $i$ for mode $k$. We will show two kinds of results in which we make the summation in Eqs. (8a) and (8b): (i) over all particles and (ii) only over the central particles. So we can clearly see which particles contribute to the compressionlike and shearlike character of the motion. In the latter case, if we sum in Eqs. (8a) and (8b) only over the central particles, we sum over all the neighbors in Eqs. (9a) and (9b) (as was recently done also in Ref. [21]), in contrast to Ref. [13] where only the nearest neighbor particle was used. This turns out to be the correct approach to get information about the compressional and shearlike properties of the modes of the central particles because the position and direction of the nearest neighbor are strongly influenced by small variations of the local environment of particle $i$. However, we noticed that if we also include the outer particles, it is important to sum in Eqs. (9a) and (9b) over all particles. Summation over only the neighbors results in a large divergence for the motion of the particles at the edge, which is not the correct result because the motion of these particles is always tangent to the hard wall.

In Fig. 7 we plot $\psi_{d}(k)$ and $\psi_{r}(k)$ as a function of the excitation frequency for $N=50$ and 300 particles (for the hard wall system with Coulomb interparticle potential). Similar results were obtained for a dipole interaction. The first column shows results when we sum over all particles, the second column is when we include only the central ones. The large values for $\psi_{r}(k)$ for all high frequencies in the first column figures indicate the shearlike character of the motion of the outer particles. The figures in the second column show that both $\psi_{d}(k)$ and $\psi_{r}(k)$ are almost zero in the high excitation frequency region. This results from the fact that we look only at the motion of the central particles and at high frequencies only the outer particles move. In the parabolic case, the lower eigenfrequency spectrum corresponds to ro-
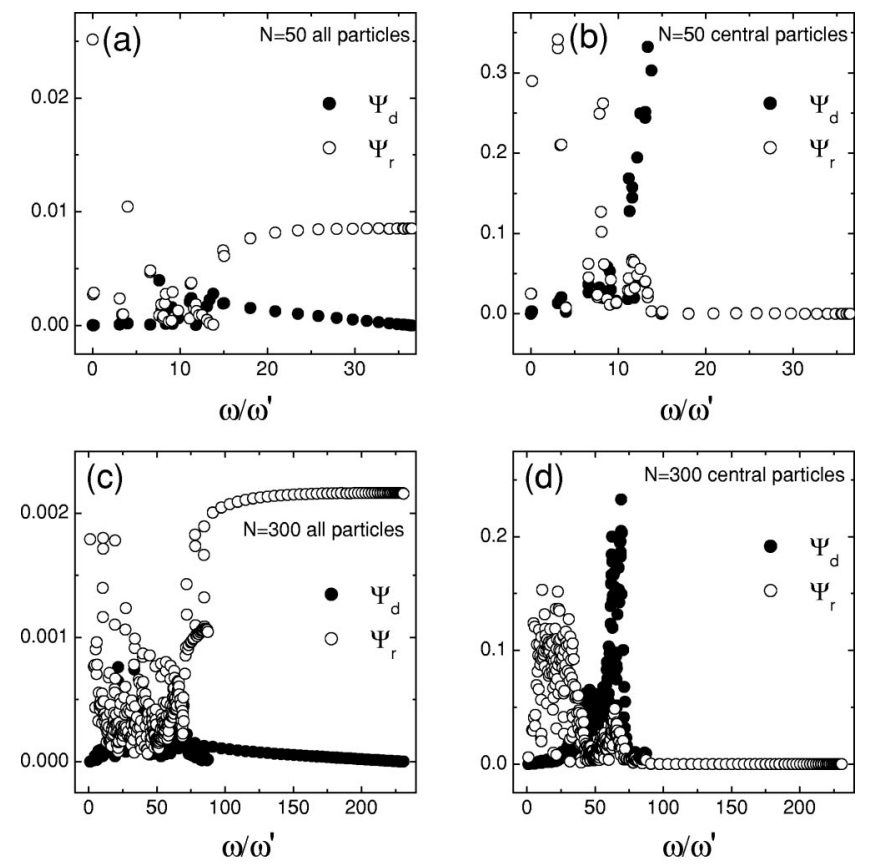

FIG. 7. The $\psi_{d}(k)$ and $\psi_{r}(k)$ as a function of the excitation frequency for $N=50(\mathrm{a}, \mathrm{b})$ and $300(\mathrm{c}, \mathrm{d})$ particles. In (a) and (c) all particles were included when calculating $\psi_{d}(k)$ and $\psi_{r}(k)$, while in (b) and (d) only the central particles were taken into account in the summation.

tational type of excitations, which are intershell rotation or vortex-antivortex-like motions of the particles which lead to practically no density fluctuations. The divergence $\psi_{d}(k)$ corresponds to compressionlike motion, and in the spectrum $\psi_{d}(k)$ can have a maximum at higher frequencies. In the hard wall confinement case, one can see that for small systems with, e.g., $N=50$ as well as for the large system with $N=300, \psi_{d}(k)$ and $\psi_{r}(k)$ have rather different values in different frequency regions and furthermore two clear separate maxima appear at the lower half of excitation spectrum. In the parabolic case, this clear separation was only present for large systems.

\section{CONCLUSION}

The configurational and spectral properties of twodimensional clusters of charged classical particles in a hard wall potential were investigated through the Monte Carlo simulation technique. A table with the packing sequences up to 50 particles was constructed for different interparticle interaction potentials. It was shown that more particles can sit at the edge in comparison with the parabolic case, and that the shell structure is much more pronounced, also for large clusters. In the case of long-range Coulomb interaction between the particles, the density of particles is increased towards the edge, while the particles are more uniformly distributed for a short-range interaction potential as e.g., for the dipole interaction.

Center-of-mass and breathing modes can be identified approximately for the central particles, while the lowest nonzero frequency corresponds with a rotation of the central 
particles as a whole (in the opposite direction to the particles at the edge). Larger frequencies correspond with many different types of intershell rotations, followed by vortexantivortex excitations. The highest frequencies involve large amplitudes for angular movement of particles at the edge, as they are almost frozen in the radial direction.

Also the shearlike and compressionlike character of the modes was investigated. A clear distinction can be made between low-frequency shearlike modes and high-frequency compressionlike modes. The highest frequency modes are again shearlike modes, corresponding with the motion of the particles at the edge.

\section{ACKNOWLEDGMENTS}

This work was supported by the Flemish Science Foundation (FWO-Vl), the Belgian Inter-University Attraction Poles (IUAP-V), the "Onderzoeksraad van de Universiteit Antwerpen"(GOA), and the EU Research Training Network on "Surface Electrons on Mesoscopic Structures." One of us (B.P.) was supported by the FWO-Vl program. Stimulating discussions with W. P. Ferreira and M. V. Milošević are gratefully acknowledged.

\section{APPENDIX A: THE EIGENFREQUENCIES OF TWO PARTICLES}

In this part, we analytically calculate the eigenfrequencies of two particles in a general confinement potential $V(r)$ $=r^{n}$. The potential energy of a 2D system of $N$ charged particles in a general $r^{n}$ confinement potential and interacting through a $1 / r^{n^{\prime}}$ potential is given by

$$
E=\sum_{i=1}^{N} \frac{1}{2} m \omega_{0}^{2} R^{2}\left(\frac{r_{i}}{R}\right)^{n}+\frac{e^{2}}{\varepsilon R} \sum_{i>j}^{N} \frac{R^{n^{\prime}}}{\left|\vec{r}_{i}-\vec{r}_{j}\right|^{n^{\prime}}},
$$

where $m$ is the mass of the particles and $\omega_{0}$ the radial confinement frequency. $n \rightarrow \infty$ corresponds to the hard wall limit. The potential energy can be written in dimensionless form using the following units for length and energy:

$$
\begin{gathered}
r_{0}=\left(e^{2} /(\varepsilon \alpha)\right)^{1 /\left(n+n^{\prime}\right)} R^{\left(n^{\prime}+n-3\right) /\left(n+n^{\prime}\right),} \\
E_{0}=\left(e^{2} / \varepsilon\right)^{n /\left(n+n^{\prime}\right)} \alpha^{n^{\prime} /\left(n+n^{\prime}\right)} R^{\left(2 n^{\prime}-n\right) /\left(n+n^{\prime}\right)}
\end{gathered}
$$

with $\alpha=m \omega_{0}^{2} / 2$. In the limit of a hard wall confinement, the length unit becomes $r_{0} \rightarrow R$ which is the radius of the hard wall, and the energy unit becomes $E_{0} \rightarrow\left(e^{2} / \varepsilon\right) / R$. In the case of parabolic confinement, i.e., $n=2$, and Coulomb interaction, i.e., $n^{\prime}=1$, we recover the previously defined units [11]: $r_{0}=\left(e^{2} / \varepsilon\right)^{1 / 3} \alpha^{-1 / 3}$ and $E_{0}=\left(e^{2} / \varepsilon\right)^{2 / 3} \alpha^{1 / 3}$. The dimensionless expression for the potential energy is given by

$$
H=\sum_{i=1}^{2} r_{i}^{n}+\frac{1}{\left|\vec{r}_{1}-\vec{r}_{2}\right|^{n^{\prime}}} .
$$

If we choose the $x$ axis through both particles, the groundstate configuration is given by
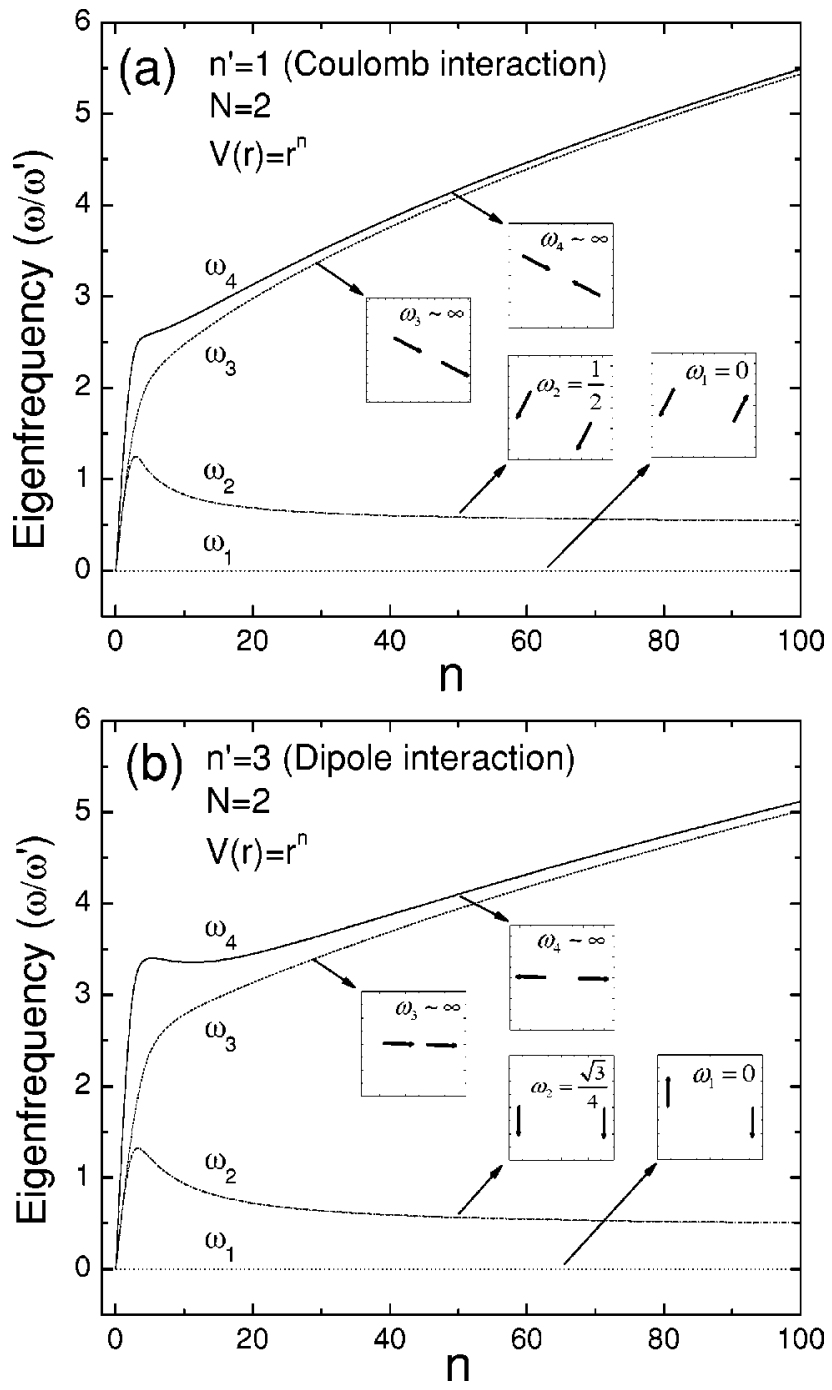

FIG. 8. The eigenfrequencies for two particles interacting through an $r^{n}$ confinement potential for (a) a Coulomb interparticle potential and (b) a dipole interaction potential. The insets show the motion of the particles and the given frequencies are for $n \rightarrow \infty$.

$$
x_{1}=\left(\frac{n^{\prime}}{n \times 2^{n^{\prime}+1}}\right)^{1 /\left(n+n^{\prime}\right)}, \quad x_{2}=-x_{1}, \quad y_{1}=y_{2}=0 .
$$

For $n \rightarrow \infty$ we find $x_{1}=1$. The eigenfrequencies are obtained by diagonalizing the dynamical matrix, resulting in

$$
\begin{gathered}
\omega_{1}^{2}=0, \\
\omega_{2}^{2}=n\left(\frac{n^{\prime}}{n \times 2^{n^{\prime}+1}}\right)^{(n-2) /\left(n+n^{\prime}\right)} \rightarrow \frac{n^{\prime}}{2^{n^{\prime}+1}}, \\
\omega_{3}^{2}=n(n-1)\left(\frac{n^{\prime}}{n \times 2^{n^{\prime}+1}}\right)^{(n-2) /\left(n+n^{\prime}\right)} \rightarrow n \frac{n^{\prime}}{2^{n^{\prime}+1}},
\end{gathered}
$$




$$
\omega_{4}^{2}=\frac{n n^{\prime}+n^{\prime 2}}{2^{n^{\prime}+1}\left(\frac{n^{\prime}}{n \times 2^{n^{\prime}+1}}\right)^{\left(n^{\prime}+2\right) /\left(n+n^{\prime}\right)}} \rightarrow n \frac{n^{\prime}}{2^{n^{\prime}+1}},
$$

where " $\rightarrow$ " means the $n \rightarrow \infty$ (i.e., hard wall) limit. Notice that in the latter limit $\omega_{3}=\omega_{4}$ diverge. The four eigenmode frequencies for Coulomb and dipole interparticle interactions are illustrated in Figs. 8(a) and 8(b).

\section{APPENDIX B: PERIODIC MOTION OF PARTICLE AT THE EDGE}

In this appendix we illustrate the motion of a particle at the edge of the hard wall boundary by solving the equation of motion. We start with the total Hamiltonian with the same potential energy term as used in our calculations, namely, $N$ particles interacting through a $1 / r^{n^{\prime}}$ potential and confined in the plane by the hard wall potential (2). In the expansion of the Hamiltonian till lowest order in the small displacements of the particles around the equilibrium ground-state positions the coordinates of the various particles are separable. Therefore it is justified to consider only the motion of a single particle. For a particle at the edge the contribution to the expanded Hamiltonian is of first order for a small displacement in the radial direction, and of second order for a small angular displacement. Therefore, we look only at the radial motion. Let us assume that the ground-state coordinates of particle 1 are given by $(-R, 0)$ and we introduce a small perturbation in the radial direction for the first particle $x=$ $-R+\Delta x$. To the lowest approximation the Hamiltonian becomes

$$
\begin{aligned}
H= & m \frac{(\Delta \dot{x})^{2}}{2}-n^{\prime} \frac{e^{2}}{\varepsilon} \sum_{i=2}^{N} \frac{\Delta x\left(-R-x_{i}\right)}{\left[\left(-R-x_{i}\right)^{2}+y_{i}^{2}\right]^{\left(n^{\prime}+2\right) / 2}} \\
& +\frac{e^{2}}{\varepsilon} \sum_{i=1}^{N-1} \sum_{j=i+1}^{N} \frac{1}{\left[\left(x_{i}-x_{j}\right)^{2}+\left(y_{i}-y_{j}\right)^{2}\right]^{n^{\prime} / 2}} .
\end{aligned}
$$

The equation of motion for the small displacement $\Delta x$ of the first particle is easily shown to be

$$
\Delta \ddot{x}=n^{\prime} \frac{e^{2}}{\varepsilon} \sum_{i=2}^{N} \frac{\left(-R-x_{i}\right)}{\left[\left(-R-x_{i}\right)^{2}+y_{i}^{2}\right]^{3 / 2}}=C,
$$

with solution

$$
\Delta x=\frac{C}{2} t^{2}+v_{o} t .
$$

This expression reminds us of the motion of a bouncing ball in the field of gravity. Its trajectory in time consists of equal pieces of interconnected parabolas. The motion starts at $t$ $=0$ at the hard wall. The particle moves in the positive direction and reaches its maximal distance from the wall at $\Delta x_{\max }=-v_{0}^{2} /(2 C)$ after $t_{\max }=-v_{0} / C$. Then the particle moves back and at $t=2 t_{\max }$ it reaches again the wall at $\Delta x$ $=0$. Now the particle is reflected elastically by the wall, it changes its momentum from $v_{0}$ to $-v_{0}$, and repeats its periodical motion. The period of this motion is given by

$$
T=2 t_{\max }=2 \sqrt{-2 \Delta x_{\max } / C},
$$

which corresponds to a frequency

$$
\omega=\frac{2 \pi}{T}=\frac{\pi}{\sqrt{-2 \Delta x_{\max } / C}} .
$$

Consequently, we have a periodic motion whose frequency depends on the amplitude of the oscillatory motion like in the case of an anharmonic oscillator. But the difference is that there is no linear regime, which is the reason that such a motion was not considered in Sec. IV. Notice that the frequency grows to infinity when the amplitude of the excitation goes to zero.

Thus in conclusion, due to the hard wall confinement potential, the particles at the edge cannot perform a harmonic motion in the radial direction. But nevertheless, these particles can perform a periodic bouncing motion against the wall which is purely anharmonic in character.
[1] E.P. Wigner, Phys. Rev. 46, 1002 (1934).

[2] C.C. Grimes and G. Adams, Phys. Rev. Lett. 42, 795 (1979).

[3] R.C. Ashoori, Nature (London) 379, 413 (1996).

[4] M. Golosovsky, Y. Saado, and D. Davidov, Phys. Rev. E 65, 061405 (2002).

[5] J.H. Chu and L. I, Phys. Rev. Lett. 72, 4009 (1994).

[6] G.A. Farias and F.M. Peeters, Solid State Commun. 100, 711 (1996).

[7] W.P. Ferreira, A. Matulis, G.A. Farias, and F.M. Peeters, Phys. Rev. E 67, 046601 (2003).

[8] R. Bubeck, C. Bechinger, S. Neser, and P. Leiderer, Phys. Rev. Lett. 82, 3364 (1999).

[9] R. Bubeck, C. Bechinger, S. Neser, and P. Leiderer, Europhys. Lett. 60, 474 (2002).
[10] I.V. Schweigert, V.A. Schweigert, and F.M. Peeters, Phys. Rev. Lett. 84, 4381 (2000)

[11] V.M. Bedanov and F.M. Peeters, Phys. Rev. B 49, 2667 (1994).

[12] N. Metropolis, A.W. Rosenbluth, M.N. Rosenbluth, A.M. Teller, and E. Teller, J. Chem. Phys. 21, 1087 (1953).

[13] V.A. Schweigert and F.M. Peeters, Phys. Rev. B 51, 7700 (1995).

[14] Y.J. Lai and L. I, Phys. Rev. E 60, 4743 (1999).

[15] L. Căndido, J.P. Rino, N. Studart, and F.M. Peeters, J. Phys.: Condens. Matter 10, 11627 (1998).

[16] M. Kong, B. Partoens, and F.M. Peeters, New J. Phys. 5, 23.1 (2003).

[17] S. Earnshaw, Trans. Cambridge Philos. Soc. 7, 97 (1842).

[18] B. Partoens and P. S. Deo, e-print cond-mat/0401207 . 
[19] M. Kong, B. Partoens, and F.M. Peeters, Phys. Rev. E 67, 021608 (2003).

[20] A. Melzer, M. Klindworth, and A. Piel, Phys. Rev. Lett. 87, 115002 (2001).
[21] A. Melzer, Phys. Rev. E 67, 016411 (2003).

[22] L. Bonsall and A.A. Maradudin, Phys. Rev. B 15, 1959 (1977); F.M. Peeters, ibid. 30, 159 (1984).

[23] R.S. Crandall, Phys. Rev. B 8, 2136 (1973). 